\title{
Methodological Limitations to the Analysis by Cicalese et al.
}

\author{
Gary M. Marsha Kevin M. Towle ${ }^{b}$ \\ ${ }^{a}$ Cardno ChemRisk, Pittsburgh, PA, USA; ${ }^{b}$ Cardno ChemRisk, San Francisco, CA, USA
}

Dear Editor,

This letter is in response to the article "An ecological study of the association between air pollution and hepatocellular carcinoma incidence in Texas" by Cicalese et al. [1], where the authors used nonparametric generalized additive logistic regression and gamma regression models to examine the association between county-level concentrations of air pollutants and hepatocellular carcinoma (HCC) incidence rates, concluding that vinyl chloride is a significant contributor to the incidence of HCC in Texas. We believe that these findings are unsubstantiated and misleading given the severe methodological limitations of the paper that we describe below.

As an ecological study, this study is limited by measuring both exposure and outcome at the population level rather than the individual level. Ecological studies are useful for generating hypotheses but are severely limited in their ability to establish causality given their many methodologic problems, including the lack of adequate data, uncontrolled confounding, specification bias, aggregation bias, multicollinearity, and temporal ambiguity [2]. In addition, several potential confounders were not considered by the authors. Therefore, the findings may be a statistical artifact of uncontrolled confounding. For example, gender, race, alcohol use, type II diabetes, and smoking are all important risk factors for HCC that were not included in the model analyses. While the authors did include an obesity term, the reported findings could have been influenced by heterogeneity and the distribution of uncontrolled risk factors at the county level.

The authors' attempt to lag exposure by using National Air Toxics Assessment (NATA) data from 1996 and 1999 for the 2000-2013 analyses and NATA data from 2002 and 2005 for the 2006-2013 analyses. However, these time points (range 1-17 years) do not allow for sufficient induction and latency periods for HCC. Specifically, a study examining vinyl chloride exposure and HCC risk reported that the median latency for HCC deaths was 48 years (range 31.5-66.6) [3]. Due to this, the exposure time points evaluated in this analysis are likely biologically irrelevant and inappropriate, as any impact on disease would not yet have been clinically diagnosed or detected.

By crudely assigning exposure values at the county level, the NATA data is a spatially diluted average value that is not an adequate representation of exposures at more refined, local levels, such as residences. Specifically, the "EPA suggests that the results of (NATA) be used cautiously... (and) (i)n many cases more localized assessments, including monitoring and modeling, may be needed to better characterize local-level risk" [4]. Regarding the 1996 NATA data, the "EPA strongly cautions that these modeling results should not be used to draw conclusions about local exposure concentrations or risk" [5]. 
Marshand Towle: Methodological Limitations to the Analysis by Cicalese et al.

This study was also biased by excluding a large percentage of counties with zero and low rates of HCC (43 and 54\% in the 2000-2013 and 2006-2013 analyses, respectively). By suppressing these counties, the authors ignored potentially important information regarding counties with low incidence rates. For example, differential misclassification may occur if excluded counties with low incidence rates of HCC were also counties with low exposure levels of vinyl chloride, which would result in a biased overestimate of the association.

The authors employed generalized additive models (GAMs) for both the logistic regression and gamma regression analyses. GAMs are utilized when there is limited information or confidence regarding the true relationship between the predictors and response. However, it is reported that consideration of potential confounders is important when using GAMs for environmental health analyses, which the authors did not fully consider [6]. Additionally, no justification was given for the selection of these modelling techniques. No model comparisons were reported to assess whether the added complexity of GAMs was required in order to achieve suitable data fit. An alternative method would be to perform diagnostic analysis of the data to investigate the functional form of possible exposure-response relationships, and then use those findings to guide modeling decisions. Further, a more appropriate and conventional methodology for incidence and mortality rate data would be the use of Poisson regression or negative binomial regression.

It should be noted that inconsistent results were reported for vinyl chloride between both the statistical models and HCC incidence rate time periods. The majority of the statistically significant findings for vinyl chloride were for the 2006-2013 analyses, which is illogical given that the latency period issue is more pronounced for this analysis. Various associations were reported for vinyl chloride based on the analysis, including negative association, positive association, parabolic association, and no association. Similar inconsistencies and changes in the direction of associations and functional forms were observed for all examined chemicals, calling into question the model choices, multicollinearity, and potential data overfitting.

\section{Disclosure Statement}

The work was supported by the Vinyl Institute, but the comments presented in the Letter to the Editor are exclusively those of the authors. The authors are employed by Cardno ChemRisk, a consulting firm that provides scientific advice to the government, corporations, law firms, and various scientific/professional organizations. G.M.M. is also professor of biostatistics, epidemiology, and clinical and translational science and director of the Center for Occupational Biostatistics and Epidemiology at the University of Pittsburgh, Graduate School of Public Health. The authors have no conflicts of interest to declare.

\section{References}

1 Cicalese L, Raun L, Shirafkan A, Campos L, Zorzi D, Montalbano M, Rhoads C, Gazis V, Ensor K, Rastellini C: An ecological study of the association between air pollution and hepatocellular carcinoma incidence in Texas. Liver Cancer 2017;6:287-296.

2 Morgenstern H: Uses of ecologic analysis in epidemiologic research. Am J Public Health 1982;72:1336-1344.

-3 Mundt K, Dell LD, Crawford L, Gallagher AE: Quantitative estimated exposure to vinyl chloride and risk of angiosarcoma of the liver and hepatocellular cancer in the US industry-wide vinyl chloride cohort: mortality update through 2013. Occup Environ Med 2017;74:709-716.

4 USEPA. National air toxics assessment, 2011 NATA: assessment results [cited 2016 Dec 26, 2016 March 30, 2018]. Available from: https://www.epa.gov/national-air-toxics-assessment/2011-nata-assessment-results.

5 USEPA. Technology transfer network, 1996 national-scale air toxics assessment. EPA strongly cautions that these modeling results should not be use [cited 2016 Feb 21, 2016 March 30, 2018]. Available from: https:// archive.epa.gov/airtoxics/nata/web/html/hapem-page2new3.html.

6 Jbilou J, El Adlouni S: Generalized additive models in environmental health: a literature review; in Luo Y (ed): Novel Approaches and Their Applications in Risk Assessment, IntechOpen, 2012. 